\title{
ATR FT-IR Absorption Enhancement of a Thin Film under the Photon-Tunneling Condition
}

\author{
Sanong Ekgasit, ${ }^{* \dagger}$ Jitraporn Vongsvivut,* and Pimthong Thongnopkun** \\ *Sensor Research Unit, Department of Chemistry, Faculty of Science, Chulalongkorn University, \\ Bangkok 10330, Thailand \\ **Burapha University, Chanthaburi IT Campus, Thamai, Chantaburi 22170, Thailand
}

\begin{abstract}
A simple, yet very powerful technique for the spectral acquisition of an extremely thin film with enhanced absorption was explored. An infrared absorption of an extremely thin film confined between media of high refractive indices was greater than that of its bulk when the spectrum was acquired under the attenuated total reflection (ATR) condition with parallel (p) polarized radiation. The absorption enhancement was not observed under perpendicular (s) polarized radiation. Theoretical investigations indicated that the absorption enhancement was proportional to the integration of the mean square evanescent field within the film. The field integration under p-polarized radiation increased, while that under spolarized radiation decreased as the thickness of the confined film became thinner. The maximum enhancement was observed when the film was sufficiently thinner than the penetration depth. The phenomena were experimentally investigated, and the results agreed very well with theoretical predictions.
\end{abstract}

(Received February 28, 2007; Accepted May 31, 2007; Published July 10, 2007)

\section{Introduction}

Attenuated total reflection Fourier transform infrared (ATR FTIR) spectroscopy is well-known for its molecular information associated with chemical moieties at the surface. The technique exploited a strong evanescent field generated under the total internal reflection (TIR) condition for probing molecular information at the surface region. To perform a surface analysis under the ATR setup, a sample of low refractive index is brought into optical contact with an internal reflection element (IRE) of high refractive index. When infrared radiation is coupled through the IRE with the angle of incidence at the IRE/sample interface greater than the critical angle, TIR occurs. Under the TIR condition, a strong electromagnetic field is generated at the interface. The field is exponentially evanescent within the sample as the distance from the interface increases. The evanescent field amplitude and its decay behavior depend strongly on the experimental conditions (i.e., angle of incidence, polarization, and frequency of the coupled radiation) and the physical property of related materials (i.e., refractive indices of the IRE and the sample). If the sample attached to the IRE is absorbing, the reflected radiation is attenuated and the reflectance becomes less than unity. ${ }^{1-4}$

ATR FT-IR spectroscopy is generally employed for the surface characterization of a semi-infinitely thick film (i.e., a film with a thickness of at least three-times the penetration depth). ${ }^{4,5}$ The absorption of an ATR spectrum is strongly dependent on the experimental conditions, and cannot be directly compared to that of the conventional transmission spectrum. The effective thickness, $d_{\mathrm{e}}$, was introduced in order to make a correlation

$\dagger$ To whom correspondence should be addressed.

E-mail: sanong.e@chula.ac.th between ATR absorption and the corresponding transmission absorption. The effective thickness is defined as the thickness of a standing film with the transmission absorbance equal to the ATR absorbance. ${ }^{1,3}$ Due to a strong evanescent field, the effective thickness is relatively small. For example, the effective thickness at $1709 \mathrm{~cm}^{-1}$, acquired by a zinc selenide ( $\mathrm{ZnSe})$ IRE with a $45^{\circ}$ angle of incidence and non-polarized radiation, equals $2.55 \mu \mathrm{m} .{ }^{4}$ The effective thickness becomes smaller when an IRE with a greater reflective index and/or a larger angle of incidence is employed. The effective thickness also becomes smaller when an absorption band at a higher frequency is investigated. The small effective thickness implies that the ATR technique may not be able to provide a sufficient sensitivity to detect a weakly absorbing material and/or a material with an extremely low concentration. However, the ATR absorbance can be increased by employing an experimental condition with a greater evanescent field amplitude and/or a great penetration depth (i.e., by using a small angle of incidence and/or an IRE of lower refractive index). However, a distortion of the absorption band may be observed due to the high refractive index dispersion at the absorption band with a strong absorption coefficient.

Since ATR FT-IR spectroscopy is a reflection-based technique, its absorption can also be increased by a factor equivalent to the number of reflections at the IRE/sample interface. The number of reflections can be calculated from the physical dimension of a flat-plate IRE by $N=(l / t) \cot \theta(l$ and $t$ are length and thickness of the IRE, respectively). ${ }^{1}$ However, increasing the aspect ratio induces any inefficient contact between the IRE and a solid sample. ${ }^{6}$ A local unevenness on the surface of the solid sample severely deteriorates the ATR spectral quality. An air gap between the IRE and the solid sample occupies the region with the strong evanescent field, and makes the evanescent field within the sample weaker. If the air 
gap is thick enough, the evanescent field decays to an insignificant level before reaching the sample, while the ATR spectrum cannot be observed. ${ }^{6}$ A pressure is normally applied onto the solid sample against the IRE in order to improve contact. However, great care must be taken, because the mirrorflat surface of the IRE can be easily damaged and/or the IRE may be broken by an excessive force.

For an ATR spectral acquisition of a thin film, the film is normally cast onto the IRE in order to achieve a good contact. However, a very thin film (i.e., a nanometers-thick film) does not exploit the entire evanescent field, since the penetration depth is substantially greater than the thickness of the film. Under the conventional ATR setup, the absorption becomes smaller as the thickness of the film becomes thinner. Grazing angle attenuated total reflection (GATR) has been developed and employed for the characterization of an organic monolayer on the surface of a silicon wafer. A grazing angle of incidence $\left(\theta \geq 65^{\circ}\right)$ is necessary for satisfying the total internal reflection criterion between the silicon substrate and the Ge IRE (i.e., the critical angle at the $\mathrm{Ge} / \mathrm{Si}$ interface equals $58.8^{\circ} ; n_{\mathrm{Ge}}=4.00$ and $\left.n_{\mathrm{Si}}=3.45\right) .{ }^{7-10}$ An optical-cavity effect in the non-contact air gap ATR technique has been employed to study of native oxide growth on the surfaces of semiconductors (i.e. silicon and GaAs). ${ }^{11,12}$

The purpose of this paper is to introduce a novel approach for enhancing the ATR absorption of an extremely thin film confined between materials of greater refractive indices. Unlike the GATR and the air-gap ATR techniques, the current technique exploits an enhanced evanescent field generated under a photon tunneling condition occurring when the distance between the IRE and a high refractive index substrate is sufficiently thin. When the gap is replaced by an absorbing medium, the corresponding ATR absorption is greatly magnified. The current investigation experimentally explores the enhanced ATR absorptions of a thin organic film confined between a Ge IRE and a Ge substrate. The observed phenomenon is theoretically explained based on the evanescent field profile under the photon-tunneling condition.

\section{Theory}

The magnitude of the reflection loss (or absorption) in an ATR spectrum is proportional to the product between the imaginary part of the complex dielectric constant and the evanescent field amplitude. The ATR absorption in absorptance unit, $A_{\overline{\mathrm{p}}}(\theta, v)$, can be expressed in terms of the experimental conditions and the material characteristics by 6,13

$$
A_{\overline{\mathrm{p}}}(\theta, v)=\frac{(2 \pi v)^{2}}{k_{\mathrm{z}, \mathrm{IRE}}(\theta, v)} \int_{0}^{\infty} \operatorname{Im}\left[\hat{\varepsilon}_{\mathrm{z}}(v)\right]\left\langle E_{\overline{\mathrm{p}} \mathrm{z}}^{2}(\theta, v)\right\rangle d z
$$

where $\theta$ is the angle of incidence, $v$ the frequency, and $\overline{\mathrm{p}}$ the polarization of the incident radiation (i.e., $\mathrm{p}$ for parallel and $\mathrm{s}$ for perpendicular polarization). $\quad \hat{\varepsilon}_{\mathrm{z}}(v)$ is the complex dielectric constant of the sample. $\left\langle E_{\mathrm{pz}}^{2}(\theta, v)\right\rangle$ is the mean square evanescent field (MSEvF) at distance $z$ from the IRE/sample interface. $k_{\mathrm{Z}, \mathrm{IRE}}(v, \theta)$ is the $\mathrm{z}$-component of the wavevector within the IRE. The $\mathrm{z}$-component wavevector can be expressed in terms of the x-component wavevector, $k_{\mathrm{x}, \mathrm{IRE}}(\theta, v)$, by $k_{\mathrm{z}, \mathrm{IRE}}(\theta, v)=\left[(2 \pi v)^{2} \varepsilon_{\mathrm{IRE}}\right.$ $\left.-k_{\mathrm{x}, \mathrm{IRE}}(\theta, v)\right]^{1 / 2}$, where $k_{\mathrm{x}, \mathrm{IRE}}(\theta, v)=(2 \pi v)^{2}\left[\varepsilon_{\mathrm{IRE}} \sin ^{2} \theta\right]$ and $\varepsilon_{\mathrm{IRE}}$ is the dielectric constant of the IRE. Detailed derivations of the MSEvF are given elsewhere. ${ }^{13,14}$

Examples of the MSEvF decay profiles within thin films are shown in Fig. 1A. When the film is semi-infinitely thick (i.e. thicker than $500 \mathrm{~nm}$ ), the field completely decays to an
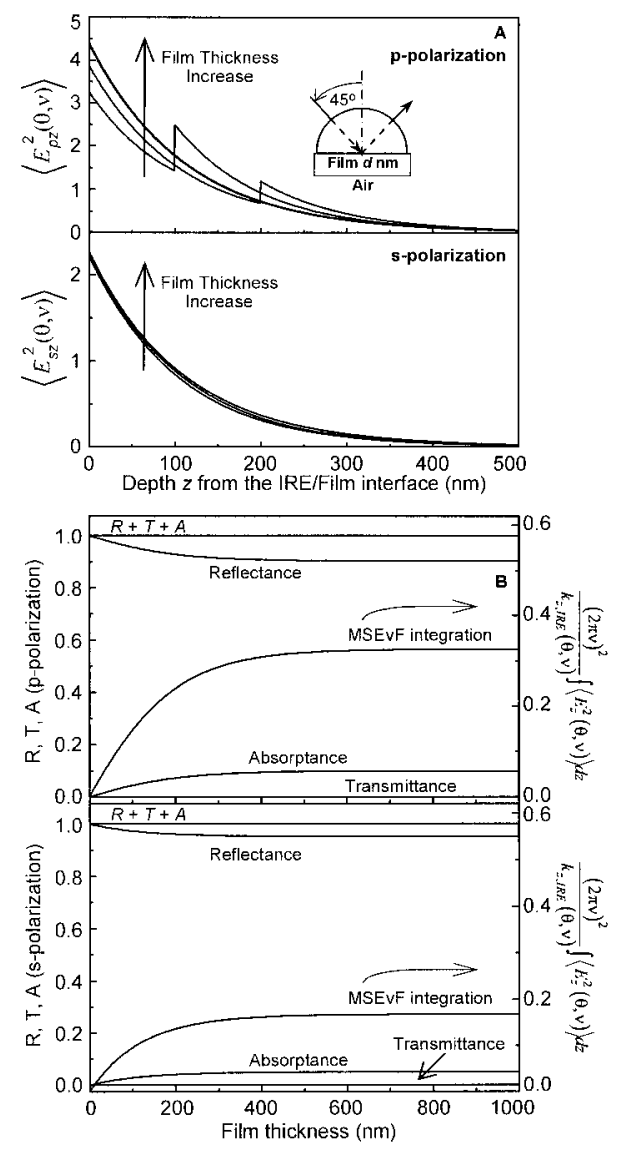

Fig. 1 (A) MSEvF decay profiles of a three-phase system $(\mathrm{Ge}$ IRE/film/air) at film thicknesses (d) of 100, 200, 500, 1000, and 5000 $\mathrm{nm}$. Since the evanescent field decays to an insignificant level near $500 \mathrm{~nm}$ from the interface, the decay profiles within the 500, 1000, and $5000 \mathrm{~nm}$ thick films are superimposed. Note: the thickness of an individual film can be observed from an abrupt change of the ppolarized MSEvF decay profile at the film/air interface. (B) MSEvF integration within the film, reflectance, transmittance, and absorptance as the function of film thickness. The simulation parameters were: $n_{\mathrm{Ge}}=4.0, \hat{n}_{\text {Film }}=1.5+0.1 i, n_{\text {Air }}=1.0, \theta=45^{\circ}, v=$ $3000 \mathrm{~cm}^{-1}$.

insignificant level within the film. As the film becomes thinner (i.e., 100 and $200 \mathrm{~nm}$ ), the MSEvF does not completely decay within the film. Instead, the field penetrates beyond the film and decays to zero within the air substrate. It should be noted that as the film becomes thinner, the p-MSEvF at the IRE/film interface decreases, while the s-MSEvF does not show any significant change. Figure $1 \mathrm{~B}$ shows that the absorption becomes weaker as the film becomes thinner, due to the smaller evanescent field integration within the film. The decrement of the absorption as the film becomes thinner is observed in both p- and s-polarized radiations.

When the same film is confined between an IRE and a material with a high refractive index (in this case a Ge IRE and a Ge plate), the MSEvF decay profile within the film changes drastically (Fig. 2A). The MSEvF decay profiles within confined films (500 and $1000 \mathrm{~nm}$ ) are the same as that within an infinitely thick film $(5000 \mathrm{~nm})$ as the fields completely decay within the films. This is due to the fact that the film thickness is greater than, or comparable to, the decay distance of the MSEvF (i.e., the penetration depth under the condition given in Fig. 2A equals $221 \mathrm{~nm}$ ). The decay distance of the MSEvF is approximately three-times the penetration depth. ${ }^{4,5}$ The 
unconventional features of the MSEvF within the confined films are the increments of the p-polarized MSEvF and the decrements of the s-polarized MSEvF as the film becomes extremely thin (i.e., 100 and $200 \mathrm{~nm}$ thick). MSEvF integrations within a film and the corresponding reflectance, transflectance and absorptance at various confined film thickness are shown in Fig. 2B. In contrast to those under the conventional ATR setup shown in Fig. 1B (i.e., the transmittance is always zero, the absorptance becomes zero while the reflectance reaches unity as the film becomes thinner), the transmittance, reflectance, and absorptance of the confined film in Fig. 2B undergo complex transitions (i.e., the transmittance and the absorptance increase, while the reflectance decreases as the film becomes thinner). The absorptance reaches a maximum before rapidly decreasing to zero as the thickness of the confined film decreases to zero.

As the thickness of the film becomes extremely thin, the increased MSEvF can not compensate for the decreased integration boundary. As a result, the p-MSEvF integration starts decreasing after reaching a maximum. Under the employed condition in Fig. 2, the absorption with a p-polarized radiation has a maximum at the thickness of a confined film of approximately $80 \mathrm{~nm}$ with absorption of 1.66-times greater than that of its bulk. Since the absorption is proportional to the MSEvF integration within the absorbing medium, the field integration can be employed as an indicator for the absorption enhancement of the confined thin film. Figure $2 \mathrm{~B}$ indicates that as the thickness of the confined film decreases, the ATR absorption with a p-polarized radiation increases in the same manner as the field integration. The MSEvF enhancement within the confined film can only be observed with p-polarized radiation. s-Polarized radiation, on the other hand, does not show any absorption enhancement, but a decrement as the thickness of the confined film decreases. It should be noted that the reflectance, transmittance, and absorptance at a certain experimental condition always follow the law of the conservation of energy (i.e., $R_{\overline{\mathrm{p}}}(\theta, v)+T_{\overline{\mathrm{p}}}(\theta, v)+A_{\overline{\mathrm{p}}}(\theta, v)=1$ ).

Although the MSEvF increment within an air gap trapped between an ATR crystal and a dielectric material (i.e., polymer film ${ }^{6}$ and silicon wafer ${ }^{15}$ ) has been mentioned, its analytical application has not yet been explored. The large enhancement of p-polarized MSEvF within an extremely thin film confined between media of greater refractive indices holds great potential for the physicochemical characterization of the film itself.

\section{Experimental}

All spectra were collected by a Nicolet Magna 750 FT-IR spectrometer (Nicolet, USA) equipped with a liquid-nitrogencooled mercury-cadmium-telluride (MCT) detector. Spectra in the mid-infrared region $\left(4000-650 \mathrm{~cm}^{-1}\right)$ were collected at a $4-\mathrm{cm}^{-1}$ spectral resolution with 512 co-addition scans. A commercial single reflection ATR accessory (the Seagull ${ }^{\mathrm{TM}}$, Harrick Scientific, USA) with a hemi-spherical Ge IRE $(25 \mathrm{~mm}$ diameter) was employed for all ATR FT-IR measurements. To collect ATR spectra of a thin organic film confined between two germanium crystals, a drop of mineral oil was placed on a $10 \times$ $10 \times 2 \mathrm{~mm}$ Ge plate. The plate was then attached to the IRE. A pressure was applied onto the Ge plate in order to decrease the thickness of the mineral oil film by squeezing the excess mineral oil trapped between two germaniums. A series of ATR spectra with a $45^{\circ}$ angle of incidence were collected as the thickness of the confined mineral oil was decreased. It should be noted that the excess amount of the mineral oil that was
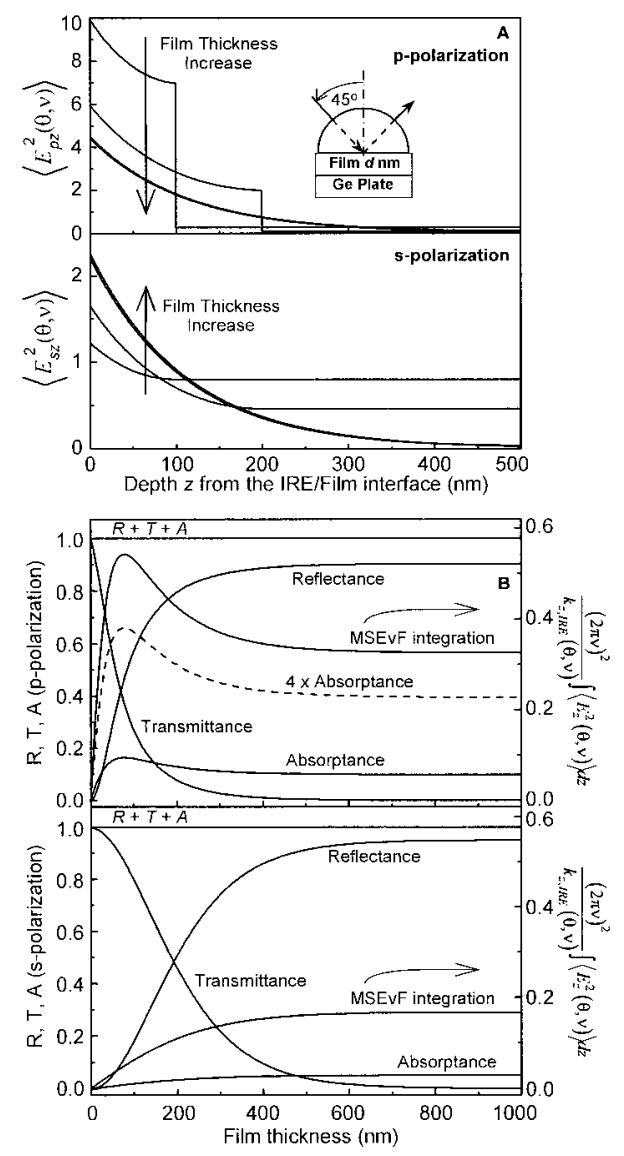

Fig. 2 (A) MSEvF decay profiles of a three-phase system (Ge IRE/film/Ge) at film thicknesses (d) of 100, 200, 500, 1000, and 5000 $\mathrm{nm}$. The thickness of the films can be observed from abrupt changes of the MSEvF profiles. (B) MSEvF integration within the film, reflectance, transmittance, and absorptance as the function of the film thickness. The simulation parameters were: $n_{\mathrm{Ge}}=4.0, \hat{n}_{\text {Film }}=1.5+$ $0.1 i, \theta=45^{\circ}, v=3000 \mathrm{~cm}^{-1}$. The photon tunneling or the frustration of the photon from the IRE across the film can be noticed via an increased transmittance as the film becomes extremely thin.

spread on the IRE outside the Ge plate does not have any contribution to the observed ATR spectra, since the illuminated area on the sensing surface of the hemispherical IRE is approximately $5 \mathrm{~mm}$ in diameter. ${ }^{16}$ An infrared circular polarizer was employed for adjusting the polarization of the coupled radiation.

\section{Results}

According to Eq. (1) and Fig. 2, the absorption of a sufficiently thin film under the confined condition can be amplified by an enhanced evanescent field. To gain an insight understanding of the anomalous absorption enhancement under the photontunneling condition, a three-phase system (i.e., Ge IRE/mineral oil film/Ge plate) was investigated. As shown in Fig. 3, the absorption of the $\mathrm{C}-\mathrm{H}$ stretching vibration observed with $\mathrm{p}$ polarized radiation increased, while that with s-polarized radiation decreased as the organic film became thinner. The absorption of the thin confined film was greater than that of the bulk. The absorption enhancement was only observed when the confined film became extremely thin. A large baseline shift in the ATR spectra was observed. The baseline at a low frequency 


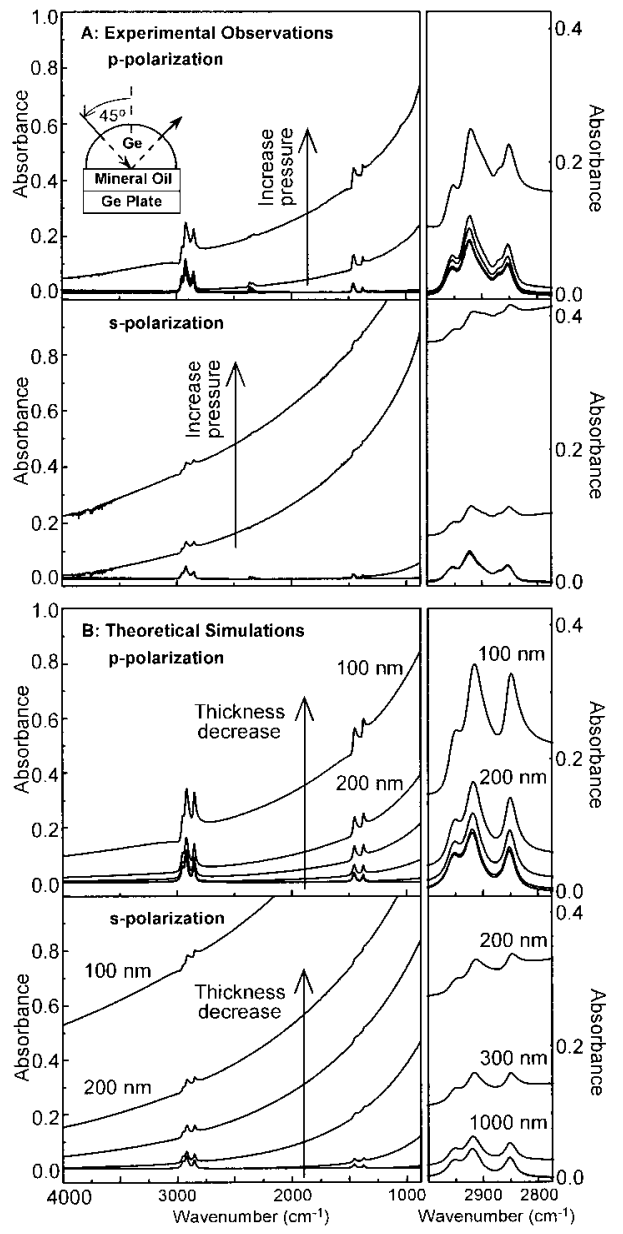

Fig. 3 (A) Experimentally observed ATR spectra of a thin mineral oil film trapped between Ge IRE and a Ge plate with p- and spolarized radiations. (B) Theoretical calculations of the investigated system (Ge IRE/mineral oil/Ge plate) with film thicknesses $(d)$ of $100,200,300,1000,2000$, and $5000 \mathrm{~nm}$. Note: both p- and s-polarized spectra were collected under the same experimental condition.

showed a greater shift than that at a high frequency. The magnitude of the baseline shift increased as the confined film became thinner. At any given thickness, the s-polarized spectrum showed a greater baseline shift than the p-polarized spectrum.

Figure 4 shows the influence of the thickness of the confined film on the baseline shift. Instead of a thin absorbing film, an air gap was trapped between the germaniums. The same patterns of the baseline shifts as shown in Fig. 3 were observed as the air gap became thinner. The transmittance profiles in Fig. 4B suggest that the baseline shift was due solely to tunneling of the coupled radiation across the thin air gap. Although the experimental condition favored total internal reflection at the $\mathrm{Ge}$ IRE/air interface, the extremely thin air gap between the Ge IRE and the Ge substrate enabled the photons to tunnel from the Ge IRE to the Ge substrate. The magnitude of the photon tunneling increased as the thickness of the air gap decreased. Since dry air is transparent in the infrared region, trapped air did not show any absorption bands (i.e., ATR absorption always equals zero) although the MSEvF integration within the air gap was very strong compared to that in Fig. 2B. The s-polarized radiation has a greater tunneling magnitude than the p-polarized radiation. A greater magnitude of the baseline shift in the spolarized ATR spectrum was due to greater tunneling at any given thickness (Fig. 4C). When the air gap was very thick,
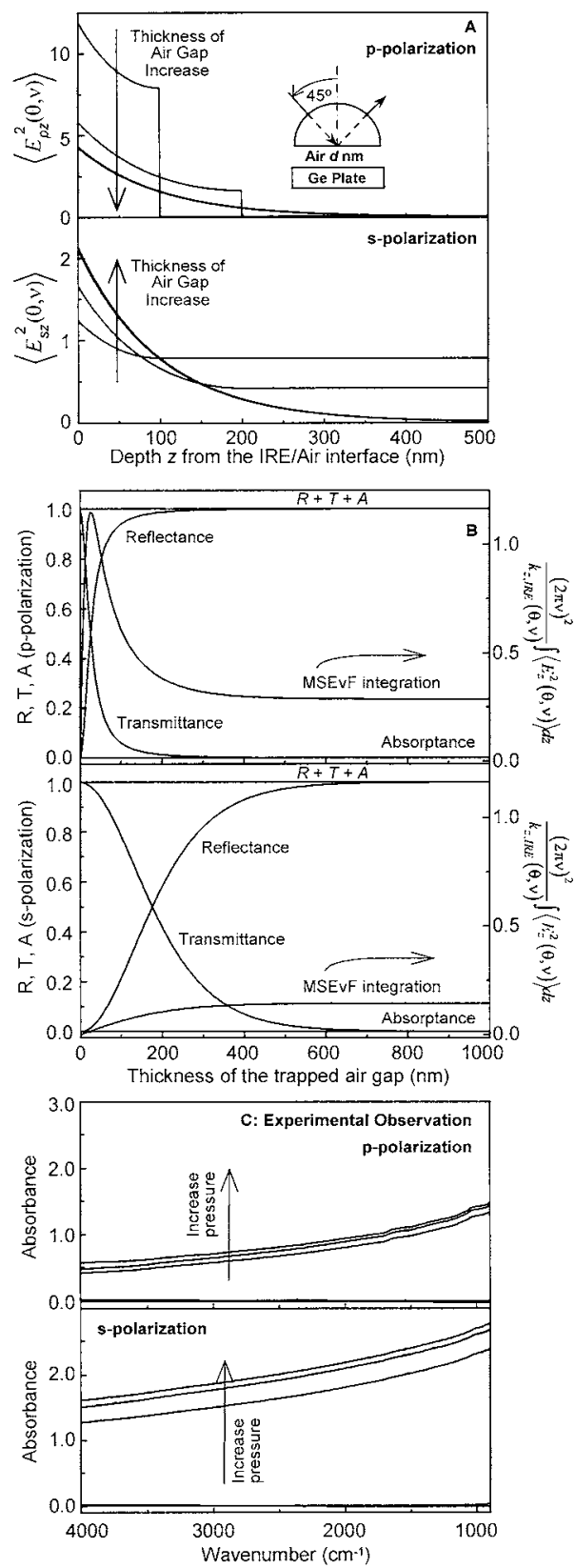

Fig. 4 (A) MSEvF decay profiles of a three-phase system $(\mathrm{Ge}$ IRE/air/Ge) at air gap thicknesses (d) of 100, 200, 500, 1000, and $5000 \mathrm{~nm}$. (B) MSEvF integration within the film, reflectance, transmittance, and absorptance as the function of the air gap thickness. (C) Experimentally observed ATR spectra of air gap trapped between Ge IRE and a Ge plate. The simulation parameters were: $n_{\mathrm{Ge}}=4.0$, $n_{\text {Air }}=1.0, \theta=45^{\circ}, v=3000 \mathrm{~cm}^{-1}$. The photon tunneling across the air gap can be experimentally observed via the baseline shift as the air gap becomes extremely thin. The extremely high evanescent field enhancement within the air gap was not exploited, since dry air is transparent in the mid-infrared region. Note: both p- and s-polarized spectra were collected under the same experimental condition.

zero absorption was observed. The photon tunneling could be recognized by the baseline shift in the experimentally observed ATR spectra as the air gap became thinner.

\section{Discussion}

The ATR absorption enhancements of the confined film with the 
p-polarized radiation and the absorption decrements with the spolarized radiation experimentally observed in Fig. 3 agree with the theoretical simulations in Fig. 2. The observed phenomena suggested that the absorption of an extremely thin film can be measured by the ATR FT-IR technique under the photon tunneling condition with an angle of incidence smaller than the grazing angle. ATR spectra of the thin confined film can be collected with absorption greater than that of the bulk. However, a p-polarized radiation must be employed in order to observe the phenomena. This is due to an optical enhancement of the p-polarized evanescent filed within the film. The presence of a high refractive index material within the decay distance of the evanescent field enhanced the p-polarized MSEvF amplitude within the confined film. The s-polarized MSEvF, on the other hand, showed a decrement under the same condition. The enhancement phenomenon was not observed when a substrate with a refractive index equals or lower than that of the film was employed.

From a theoretical point of view, the experimental conditions given in Figs. 2 and 3 satisfy the TIR condition (i.e., the refractive index of the IRE is greater than that of the film and the angle of incidence is greater than the critical angle). Under the conventional ATR setup, the transflectance $T(\theta, v)$ always equals zero (Fig. 1B). If the film is absorbing, the reflectance is less than unity due to attenuation by absorption of the film. The absorption becomes smaller as the film becomes thinner. The absorption due to an interaction of the material and the evanescent field is the sole cause for reflection loss under the conventional ATR setup.

Under the confined condition, a baseline shift occurs as the thickness of the confined film becomes sufficiently thin. The baseline shift is due to the photon tunneling across the film from the Ge IRE to the Ge substrate. Tunneling could be theoretically noticed from the increment of the transmittance as the film becomes thinner than the decay distance of the MSEvF (Fig. 2B). Photon tunneling is an additional cause for reflection loss in an ATR spectrum of a confined film. The greater is the tunneling-transmitted photon, the larger is the baseline shift. Due to the shallower penetration depth at a high frequency, the magnitude of the photon tunneling at a high frequency is smaller than that at a low frequency. Under the experimental conditions given in Fig. 1, the penetration depth at $3000 \mathrm{~cm}^{-1}$ equals $221 \mathrm{~nm}$, while that at $1000 \mathrm{~cm}^{-1}$ is $664 \mathrm{~nm}$. As a result, a smaller baseline shift at a high wavenumber and a greater shift at a low wavenumber in the same spectrum are observed. At any given film thickness, a greater shift was observed in the spolarized spectrum due to a greater tunneling transmittance (Figs. 2B and 4B). This new technique holds great potential applications for the physicochemical characterizations of an extremely thin film, especially a nanometers-thick film.

\section{Conclusions}

An ATR absorption enhancement of an extremely thin film under the photon-tunneling condition was experimentally observed and theoretically explained. When a nanometers-thin film was confined between media of greater refractive indices, its ATR absorption was increased by the enhanced evanescent field. The absorption of the confined film could be greater than that of the thick film or the bulk. However, the enhancement was only observed under p-polarized radiation. Under the confined condition, the maximum possible evanescent field amplitude was exploited.

\section{Acknowledgements}

The authors gratefully acknowledge support from Chulalongkorn University through the University Research Unit Program and the National Nanotechnology Center (Nanotec), the National Science and Technology Development Agency (NSTDA).

\section{References}

1. N. J. Harrick, "Internal Reflection Spectroscopy", 1987, Harrick Scientific Corporation, New York.

2. F. de Fornel, "Evanescent Waves from Newtonian Optics to Atomic Optics", 2001, Springer, Berlin.

3. M. W. Urban, "Attenuated Total Reflectance Spectroscopy of Polymers: Theory and Practice", 1996, American Chemical Society, Washington, D.C.

4. F. M. Mirabella, Jr., in "Internal Reflection Spectroscopy: Theory and Applications", ed. F. M. Mirabella, Jr., 1993, Marcel Dekker, New York, 17 - 52.

5. F. M. Mirabella, Jr., Polym. Sci. Polym. Phys. Ed., 1983, 21, 2403.

6. S. Ekgasit and A. Padermshoke, Appl. Spectrosc., 2001, 55, 1352.

7. M. Milosevic, S. L. Berets, and A. Y. Fadeev, Appl. Spectrosc., 2003, 57, 724.

8. N. Rochat, A. Cahbli, F. Bertin, C. Vergnaud, P. Mur, S. Petitdidier, and P. Besson, Mater. Sci. Eng., B, 2003, 102, 16.

9. T. Lummerstorfer and H. Hoffmann, Langmuir, 2004, 20, 6542.

10. T. Lummerstorfer, C. Sohar, G. Friedbacher, and H. Hoffmann, Langmuir, 2006, 22, 18.

11. N. Nagai, Y. Izumi, H. Ishida, Y. Suzuki, and A. Hatta, in Proceedings of 11th International Conference of Fourier Transform Spectroscopy, ed. J. A. De Haseth, 1998, AIP, Washington, D.C., 581 - 585.

12. N. Nagai and H. Hashimoto, Appl. Surf. Sci., 2001, 172, 303.

13. S. Ekgasit, C. Thammacharoen, F. Yu, and W. Knoll, Anal. Chem., 2004, 76, 2210.

14. W. N. Hansen, J. Opt. Soc. Am., 1968, 58, 380.

15. Y. Nishikawa, Appl. Spectrosc., 1999, 53, 1054.

16. S. Ekgasit and P. Thongnopkun, Appl. Spectrosc., 2005, 59, 1236. 